\title{
Hodgkin's disease and birth outcome: a Danish nationwide cohort study
}

\author{
V Langagergaard", I,2, E Horvath-Puho', M Nørgaard', B Nørgård' and HT Sørensen' \\ 'Department of Clinical Epidemiology, Aarhus University Hospital, Ole Worms Allé 150, Aarhus C DK-8000, Denmark; ${ }^{2}$ Department of Epidemiology, \\ Institute of Public Health, Aarhus University, Vennelyst Boulevard 6, Aarhus C DK-8000, Denmark
}

\begin{abstract}
In a Danish nationwide cohort study of 292 births from 1973 to 2002 in women with Hodgkin's disease (HD), we compared birth outcome with 14042 births from a cohort of mothers without cancer. We found no substantially increased risk of preterm birth, low birth weight at term, or stillbirth and no difference in proportion of male newborns for 192 children of women with HD before pregnancy. The prevalence odds ratio (POR) for congenital abnormalities was I.7 (95\% confidence interval (Cl): 0.9-3.I). Among I5 newborns of mothers diagnosed during pregnancy, the POR of preterm birth was 26.6 ( $95 \% \mathrm{Cl}: 8.5-83.0)$, but five out of the eight preterm deliveries among these women were elective. We found no substantially increased risk of adverse birth outcome among 85 newborns of women diagnosed within 2 years postpartum, though effect estimates were imprecise. The overall findings are reassuring, they cannot exclude the possibility of an increased risk of congenital abnormalities for newborns of women diagnosed with HD before pregnancy.

British Journal of Cancer (2008) 98, 183-188. doi: 10.1038/sj.bjc.6604I26 www.bjcancer.com

Published online 4 December 2007

(c) 2008 Cancer Research UK
\end{abstract}

Keywords: Hodgkin's disease; epidemiology; pregnancy; birth outcome; cohort study

Hodgkin's disease (HD) can affect women of childbearing age (Fisher and Hancock, 1996). Advances in its treatment have led to an overall 5-year relative survival of more than $80 \%$ (Melbye and Adami, 2002). However, there is concern (Swerdlow et al, 1996) that treatment may affect future pregnancies either by direct effects on the reproductive tract or by causing mutations in germ cells (Nagarajan and Robison, 2005). Furthermore, cancer treatment administered in the first trimester may be teratogenic (Fisher and Hancock, 1996), while detriments in maternal well-being may influence pregnancies in women with preclinical HD (Koren et al, 1996).

Nevertheless, data concerning birth outcome in women with previous HD are sparse and consist mainly of case series (McKeen et al, 1979; Andrieu and Ochoa-Molina, 1983; Green and Hall, 1988; Aisner et al, 1993; Brierley et al, 1998). A few of these case series, which included birth outcome in 15-54 women found a high prevalence of adverse outcome. Green and Hall (1988) reported 4 stillbirths among 28 pregnancies (14.3\%) in women with previous HD, while McKeen et al (1979) reported 6 premature/low birth weight children $(15.0 \%)$ and 3 with major congenital abnormalities (7.5\%) among 40 pregnancies. The remaining studies found little, if any, detrimental effect on birth outcome among women capable of becoming pregnant (Andrieu and Ochoa-Molina, 1983; Aisner et al, 1993; Brierley et al, 1998). Likewise, a few case series found normal birth outcome in women diagnosed with HD during or shortly after pregnancy (Woo et al, 1992; Anselmo et al, 1999; Aviles and Neri, 2001).

\footnotetext{
*Correspondence: Dr V Langagergaard; E-mail: Vivian.Langagergaard@stab.rm.dk

Received 15 August 2007; revised 12 November 2007; accepted 14 November 2007; published online 4 December 2007
}

We examined the risk of adverse birth outcomes in a Danish nationwide cohort of women with HD before or during pregnancy, or within 2 years after delivery and compared them with those in a cohort of pregnant women without cancer.

\section{MATERIALS AND METHODS}

We used the Danish Cancer Registry, which has covered all incident cancers in Denmark since 1943, classified according to the International Classification of Diseases (ICD-7) (Storm et al, 1997), to trace all women with a diagnosis of HD (ICD-7 code 201). Information included the civil registration number of the woman, date of diagnosis, and radiation treatment administered within 4 months of diagnosis.

Since 1 January 1973, all births in Denmark have been registered in the Danish Medical Birth Registry (Knudsen and Olsen, 1998). Data are obtained from birth notifications, which are completed by midwives (who attend all births, including home births, in Denmark). The main variables in the Birth Registry are gestational age, birth weight, parity, stillbirth, place of birth, and the civil registration number of the mother and child (which encodes sex and date of birth and is assigned to all live-born children and new residents; Frank, 2000).

Using the civil registration number, we linked the Cancer Registry data with the Birth Registry to establish a cohort of all Danish women with a diagnosis of HD in, 1970-2002, and who gave birth in 1973-2002. Women were included if they were diagnosed with HD before pregnancy, during the pregnancy, or until 2 years postpartum. We restricted all analyses to singleton births, since multiple births have been associated with an adverse birth outcome (Pinborg et al, 2004). 
For each birth by a woman with HD, 50 comparison births matched by month and year of the birth, by county of mother's residence, and born to 50 different women who were not diagnosed with any cancer before, during, or within 2 years after the pregnancy were selected from the Birth Registry. If fewer than 50 births fulfilled the matching criteria, we used all the available births. If more than 50 comparison births were eligible after matching, we selected a random subset of 50 births. On average, 48 comparison births were selected for each exposed birth.

The outcome data collected from the Birth Registry included preterm birth (birth before 37 completed weeks of pregnancy), low birth weight at term $<2500$ g with $\geqslant 37$ completed weeks), stillbirth (delivery of a dead foetus at $\geqslant 28$ completed weeks of pregnancy), male proportion of newborns, and birth weight. The potential confounders included maternal age, parity, gestational age, and calendar period of the birth. For live-born children, data on congenital (including chromosomal) abnormalities, diagnosed during the first year of life were collected from the National Hospital Discharge Registry, covering all hospital discharge diagnoses since 1977 and outpatient visits since 1995 (Andersen et al, 1999). Thus, data on congenital abnormalities applied to births from 1977 to 2002 . The data include the civil registration number, dates of admission and discharge, and up to 20 discharge diagnoses, (ICD-8 before 1994 and ICD-10 from 1994 onwards; Andersen et al, 1999). The codes for congenital (including chromosomal) abnormalities were 740.00-759.99 in ICD-8 and
Q0.00 to Q99.9 in ICD-10. Diagnoses of congenital dislocation of the hip and undescended testis were excluded because of their poor validity (Larsen et al, 2003).

Birth weights $\geqslant 7000 \mathrm{~g}$ probably reflected coding errors and were excluded, as were births with a gestational age below 20 or over 44 weeks. Owing to a coding change in the Birth Registry in 1978 , there were more missing data on gestational age for the years 1978 - 1981 than for other years (mean 22.6\% missing for 1978-1981, compared with $0.8 \%$ in $1973-1977$ and $1.2 \%$ in $1982-2002$ ). Births without data on gestational age were excluded from the study $(N=20$ in the exposed and 698 in the comparison cohort).

We classified the births of women with HD into three groups: group 1 included the first birth after an HD diagnosis (that is, women who were diagnosed before pregnancy). Group 2 included the births by women diagnosed with HD during pregnancy (that is, diagnosed between the first day in the last menstruation until the date of birth). Group 3 included births by women who were diagnosed with HD after delivery (that is, diagnosed between the day after the delivery until 2 years later). If a woman gave birth more than once in this 2-year period, only the last birth before the HD diagnosis was included based on the assumption that the preclinical cancer would be more likely to affect the birth closest to the time of diagnosis.

For all three groups, we computed the difference between proportions of male newborns of mothers with $\mathrm{HD}$ and comparison mothers.

Table I Characteristics of births by women with Hodgkin's disease and by women in the comparison cohort

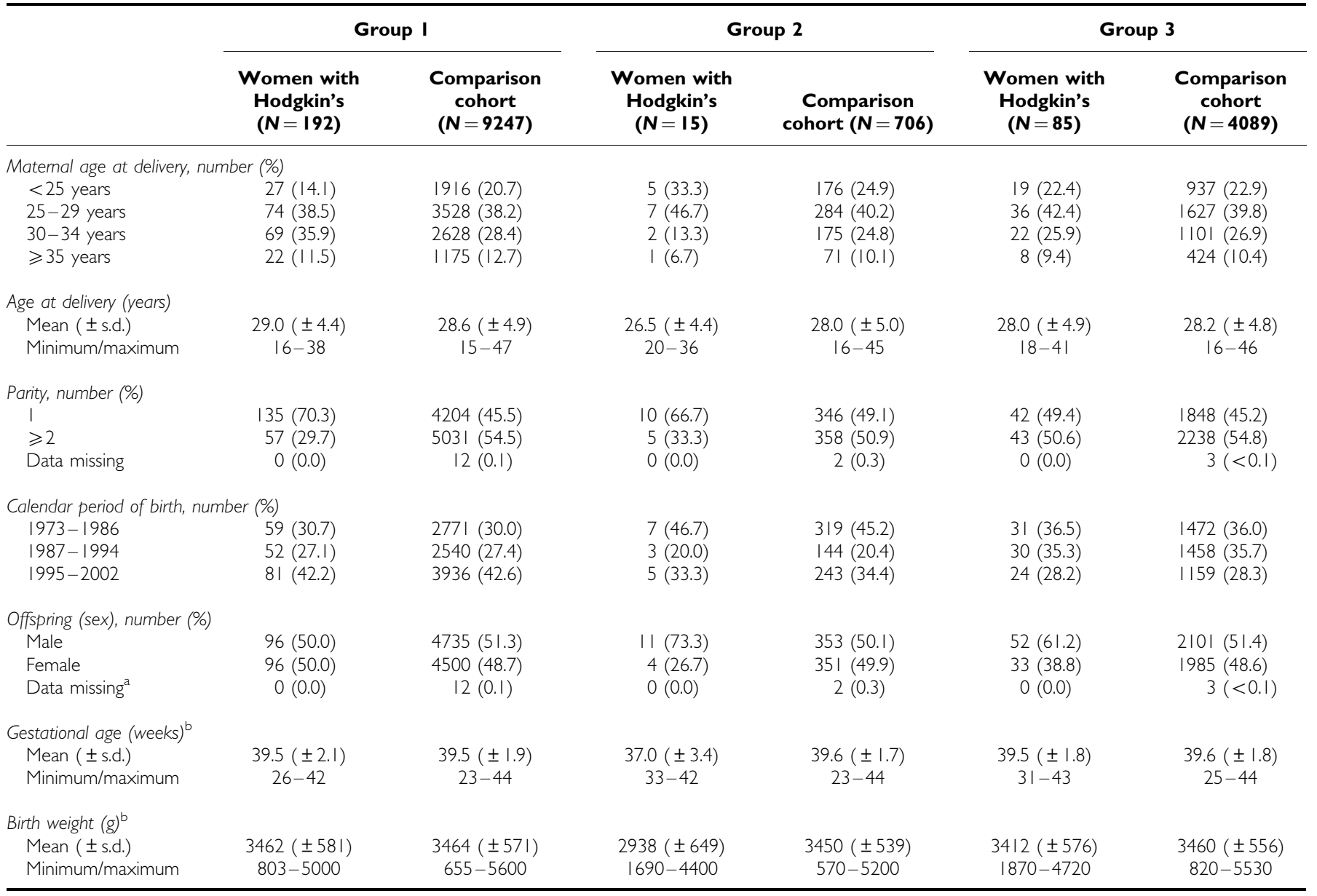

Abbreviation: CPR-number = civil registration number. Group I: births by women diagnosed with Hodgkin's disease before pregnancy. Group 2: births by women diagnosed

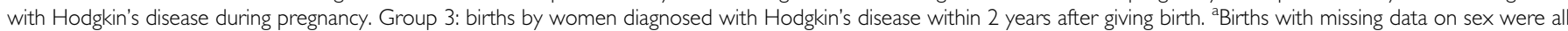
stillbirths who had no CPR-number. 'Stillborn babies were excluded from the analyses of mean gestational age and mean birth weight. 
We computed prevalence odds ratios (PORs) as estimates of the relative risks with associated 95\% confidence intervals (95\% CIs) for preterm birth, low birth weight at term, stillbirth, and congenital abnormalities. The PORs were controlled for month and year of birth and county of mother's residence by matching. We used unconditional logistic regression analysis to further adjust for maternal age and parity. We also included the calendar period of the birth (1973-1986, $1987-1994$, and 1995-2002), as an independent variable in the model. Although there was no change in the risk estimates when calendar period of birth was included in the model, we kept the variable in the model. Stillborn children were excluded from the analyses of preterm birth, low birth weight at term, and congenital abnormalities.

To examine whether sex of the child or maternal radiotherapy modified the POR estimates for births in group 1, we repeated the analyses in strata of boys and girls and strata of births of women who were treated with radiotherapy and women who were not. Furthermore, to examine whether calendar period of HD diagnosis modified the POR estimates for births in group 1, we repeated the analyses in different calendar periods of HD diagnosis (1981-1990 and 1991-2000), using 1970-1980 as reference. We used the Wald test to evaluate the homogeneity of the POR estimates for congenital abnormalities in 1981-1990 and 1991-2000. The low count of outcome events in groups 2 and 3 precluded stratified analyses.

We used linear regression to estimate differences in mean birth weight, while controlling for maternal age, parity, gestational age, and calendar period of birth. Stillborn children were excluded from these analyses.

The study was approved by the Danish Data Protection Agency (record no. 2003-41-2833). All analyses used SAS software, version 8.2. The SAS procedures used were PROC FREQ, PROC MEANS, PROC LOGISTIC, and PROC GLM.

\section{RESULTS}

In total, we identified 292 singleton births delivered by women with HD and selected 14042 singleton births for the comparison cohort. The characteristics of births in the three groups and their comparison births are shown in Table 1. Of the 292 births by women with HD, 192 occurred in group 1. The median number of days from the time of diagnosis until pregnancy (that is, the first day in the last menstruation) was 1824 days (range: 279-7877 days). The majority of women (76\%) in group 1 were $\geqslant 20$ years of age at time of HD diagnosis (data not shown) and the mean age at delivery was 29.0 years. Seventy percent of the women gave birth for the first time. Group 2 included 15 births (eight women were diagnosed in the second trimester and seven in the third). In this group, the mean age at delivery was 26.5 years and $66.7 \%$ gave birth for the first time. Group 3 included 85 births. The median number of days from date of giving birth until date of cancer diagnosis was 321 days (range: 6-709 days). The mean age at delivery was 28.0 years, and $49.4 \%$ of the women gave birth for the first time.

The prevalence of male newborns of women with HD in group 1 was $50.0 \%$, compared with $51.3 \%$ among the matched comparison mothers (difference $=-1.3 \%, 95 \% \mathrm{CI}$ : -8.4 to 5.8 ). The corresponding findings were 73.3 vs $50.1 \%$ (difference $=23.2 \%, 95 \%$ CI: $5.1-45.6$ ) for group 2, and 61.2 vs $51.4 \%$ (difference $=9.8 \%, 95 \%$ CI: -0.7 to 20.3 ) for group 3.

Table 2 shows PORs for preterm birth, low birth weight at term, stillbirth, and congenital abnormalities for newborns in all three groups. For group 1, there was no increased risk of preterm birth or low birth weight at term. We found only 1 stillbirth among 192 births, corresponding to a POR of 2.0 (95\% CI: 0.3-15.4). The POR for congenital abnormalities was 1.7 (95\% CI: $0.9-3.1)$. In groups 2 and 3 , there were no children with low birth weight at term and no stillbirths. The POR of preterm birth in group 2 was 26.6 (95\% CI: 8.5-83.0). However, five of the eight preterm deliveries among women with HD were elective preterm deliveries. There was 1 child with a congenital abnormality among 13 births in group 2 $(\mathrm{POR}=2.7,95 \% \mathrm{CI}: 0.3-22.8)$ and 4 children with congenital abnormalities among 78 births in group 3 (POR $=1.6,95 \%$ CI: $0.6-4.5)$. The specific types of congenital abnormalities identified in children of women with HD in groups 1,2 , and 3 are listed according to affected organ system in Table 3.

Table 4 shows the birth outcomes in group 1, stratified according to maternal radiotherapy (yes/no) and three calendar periods of HD diagnosis. Stratification suggested a slightly lower risk (except for stillbirths) of adverse birth outcomes in women

Table 2 Prevalence odds ratios of birth outcome in women with Hodgkin's disease

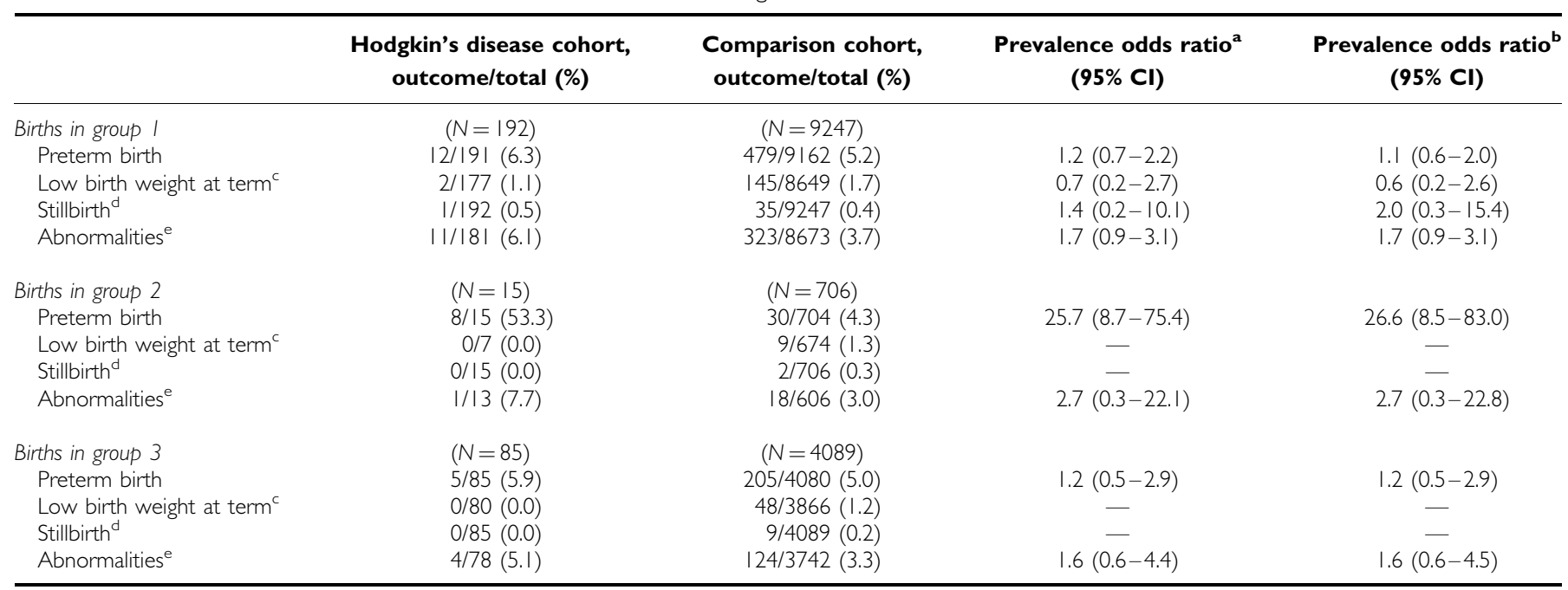

Abbreviation: $\mathrm{Cl}=$ confidence interval. Group I: birth outcome in women diagnosed with Hodgkin's disease before pregnancy. Group 2: birth outcome in women diagnosed with Hodgkin's disease during pregnancy. Group 3: birth outcome in women diagnosed with Hodgkin's disease within 2 years postpartum. ${ }^{\mathrm{a} C o n t r o l l e d ~ f o r ~ m o n t h ~ a n d ~ y e a r ~ o f ~ t h e ~}$ birth and maternal county of residence (by matching). ${ }^{b}$ Further adjusted for maternal age $(<25,25-29,30-34$, and $\geqslant 35$ years $)$ and parity $(I$ and $2+)$ by logistic regression. Calendar period of the birth (1973-1986, 1987-1994, and 1995-2002) was also included as an independent variable in the model. ${ }^{~}$ Preterm births were excluded from the analyses of low birth weight at term. ${ }^{\mathrm{d} S}$ Sillborn babies were excluded from the analyses of preterm birth, low birth weight at term, and congenital abnormalities. ${ }^{\mathrm{e}}$ Data on congenital abnormalities included births from 1977 to 2002. 
Table 3 Congenital abnormalities diagnosed during the first year of life in children of Hodgkin's disease patients

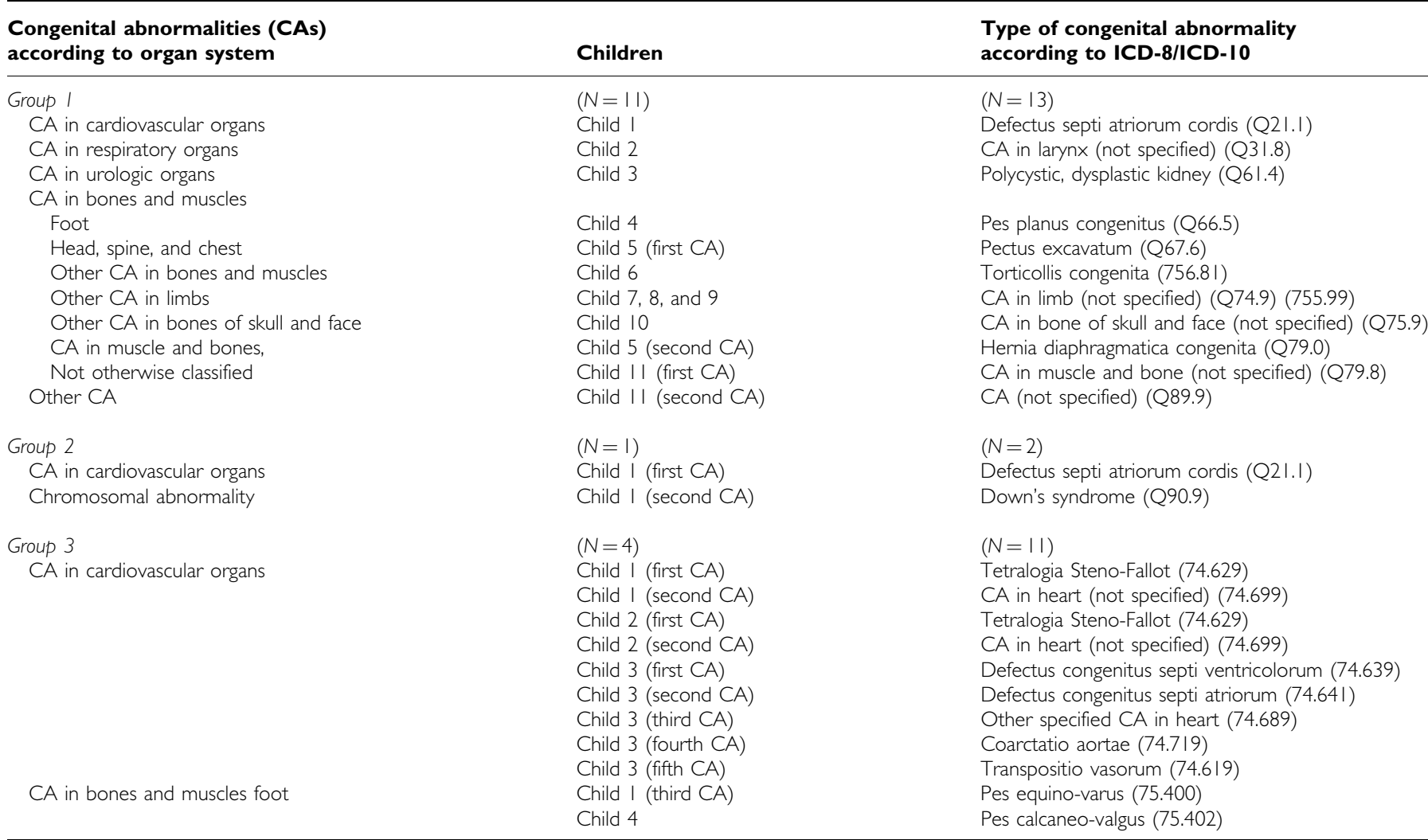

Group I: birth outcome in women diagnosed with Hodgkin's disease before pregnancy. Group 2: birth outcome in women diagnosed with Hodgkin's disease during pregnancy. Group 3: birth outcome in women diagnosed with Hodgkin's disease within 2 years postpartum.

who had received radiotherapy. Furthermore, we found that the POR for congenital abnormalities increased with calendar time of HD diagnosis (Wald test of the homogeneity of the POR estimates for 1981-1990 and 1991-2000, with 1970-1980 as reference; $P=0.25)$. Stratification according to sex of newborns did not substantially change the estimates (data not shown).

The multiple linear regression analyses showed that newborns in all three groups had nearly the same mean birth weight as newborns in the comparison cohort (data not shown).

\section{DISCUSSION}

This nationwide cohort study on the relation between maternal HD and adverse birth outcome did not show any increased risk of preterm birth or low birth weight at term, and no substantial increased risk of stillbirth in women with previous HD. However, we cannot rule out the possibility of a higher risk of congenital abnormalities for newborns of these women.

The accuracy of our risk estimates depends on several factors. The main strength of the study is the underlying uniform healthcare system, with complete registration of cancers and births and complete follow-up on congenital abnormalities diagnosed during the first year of life, allowing for a population-based design. Information on congenital abnormalities in the Hospital Discharge Registry is generally of high quality, with an $85 \%$ correct coding rate (Larsen et al, 2003). The quality of most outcome variables in the Birth Registry is high, but gestational age is subject to some misclassification (Kristensen et al, 1996) but is probably nondifferential between HD and cancer-free mothers.

Although our study population was large compared with other studies, a limitation is the small number of outcomes. Furthermore, the data lacked clinical detail on radiation fields, doses, and duration of treatment, and we had no information on chemotherapy or disease stage; radiotherapy details (yes/no) from the Cancer Registry may be inaccurate, because they are not routinely validated. However, a study of childhood cancer survivors reported that 97 out of 110 patients treated with radiotherapy $(88 \%)$, and 78 out of 79 patients not treated with radiotherapy (99\%) were correctly coded in the Registry (Ross et al, 2003).

Women with early-stage HD, which is often located above the diaphragm, were probably more likely than women with more advanced stages, to receive radiotherapy, since the typical treatment of early-stage disease in our study period has been either radiation alone (with minimal effect on the gonads in case of supradiaphragmatic location), or a few series of combination chemotherapy followed by radiation. In contrast, later stages of HD have typically been treated with six series of combination chemotherapy and only rarely radiotherapy. Thus, an uneven distribution of stage could have biased our results and may explain our finding of a lower risk of adverse birth outcomes for women treated with radiotherapy, compared with those who were not.

Fetal abnormalities may lead both to miscarriage (Yusuf and Naeem, 2004) and to induced abortion, but we had no data on these outcomes. Thus, selection bias could have occurred if women with HD had more miscarriages and induced abortions related to fetal abnormalities than did comparison mothers. Such bias would lead us to underestimate the risk of congenital abnormalities in newborns of women with HD.

It has been suggested that mutagenic exposure of germ cells (that is, chemotherapy or radiation) may decrease the proportion of male newborns in female survivors of cancer due to sex-linked lethal mutations (Nagarajan and Robison, 2005). Our data, 
however, showed no substantial decrease in the male proportion of newborns in group 1, indicating that earlier treatment for $\mathrm{HD}$ is not a risk factor for early male abortion. For newborns in group 2, there was an increase in the male proportion compared with newborns of comparison mothers. This finding is surprising and may be due to chance. The male proportion of newborns in Denmark is approximately $51.2 \%$ (Hansen et al, 1999).

We believe that our study is the first to estimate relative risks for congenital abnormalities among newborns of women with HD. The increased risk estimates found after diagnoses during or shortly after pregnancy were imprecise. However, it is relevant that teratogens increase the rate of specific abnormalities but not all abnormalities (Mitchell, 2000), and we were unable to evaluate the risk of specific abnormalities. Small cohort studies can detect only large increases in the risk of specific congenital abnormalities and are limited in their ability to provide an assurance of safety. Our finding of a higher risk of abnormalities for newborns of women with HD from 1991 to 2000 (before their pregnancy) may be a diagnostic bias caused by a recently increased interest in such risk after maternal cancer treatment.

Overall, our findings are in line with the existing studies. Two cohort studies found no substantial increased risk of low birth weight and no congenital abnormalities among newborns of 15 women with previous HD (Janov et al, 1992) and no increased risk of preterm birth, low birth weight, stillbirth, congenital abnormalities, or chromosomal abnormalities among 49 children of 16 women and 11 men who had previously been treated for HD (Swerdlow et al, 1996). Both studies were compared with birth outcomes in the general population. Another cohort study compared 52 births of 29 women previously treated for HD with births of siblings of the women (Holmes and Holmes, 1978). There was no overall increased risk of adverse birth outcome (that is, congenital abnormalities and stillbirths combined) for HD patients and no increased risk associated with radiation treatment alone (supra- or infradiaphragmatic), whereas women treated with both chemotherapy and radiation were more likely to have an adverse birth outcome $(P=0.047)$. These three studies, however, were all based on small study population and did not control for potential confounders.

Recently, a large cohort study of female survivors of childhood cancer found that $19.2 \%$ of 337 women with childhood HD had a preterm birth compared with $12.6 \%$ among sibling controls (Signorello et al, 2006). Another study reported 11 stillbirths among 729 births of female survivors of childhood HD, corresponding to a relative risk of 1.6 (95\% CI: 0.64-4.03) (Green et al, 2002). We found no increased risk of preterm birth and only 1 stillbirth among 192 women, of whom more than $75 \%$ had been diagnosed with HD in adulthood ( $\geqslant 20$ years of age at diagnosis).

The 26-fold increased risk of a preterm delivery for women diagnosed with HD during pregnancy reflected a higher rate of elective early delivery, probably to allow an early start of cancer therapy. This finding is consistent with another study on pregnant women with HD (Smith et al, 2001) which identified 172 cases of HD diagnosed from 9 months preceding delivery until 12 months after delivery and found relative risks of 2.4 (95\% CI: 1.6-3.5) for prematurity and 3.6 (95\% CI: $1.5-8.9)$ for very low birth weight. The authors suggested that these findings reflected a higher rate of elective early deliveries to allow initiation of therapy. In contrast, a historical cohort study, which included 40 births of women who were pregnant between 9 months before and 3 months after their first treatment for HD, reported no increased risk of preterm birth or induced deliveries (Lishner et al, 1992). Furthermore, it indicated no difference in mean birth weight compared with controls and no increased risk of stillbirths, and overall, its findings corroborate our data, except for preterm births.

The overall findings of this nationwide cohort study are reassuring, but we cannot rule out the possibility of an increased risk of congenital abnormalities in offspring of women diagnosed with HD before pregnancy. 


\section{ACKNOWLEDGEMENTS}

The study received financial support from the Danish Cancer Society; the Danish Cancer Research Foundation; the Western Danish Research Forum for Health Sciences; Ingeborg and Leo

\section{REFERENCES}

Aisner J, Wiernik PH, Pearl P (1993) Pregnancy outcome in patients treated for Hodgkin's disease. J Clin Oncol 11: 507-512

Andersen TF, Madsen M, Jorgensen J, Mellemkjoer L, Olsen JH (1999) The Danish National Hospital Register. A valuable source of data for modern health sciences. Dan Med Bull 46: 263-268

Andrieu JM, Ochoa-Molina ME (1983) Menstrual cycle, pregnancies and offspring before and after MOPP therapy for Hodgkin's disease. Cancer 52: $435-438$

Anselmo AP, Cavalieri E, Enrici RM, Pescarmona E, Guerrisi V, Paesano R, Pachi A, Mandelli F (1999) Hodgkin's disease during pregnancy: diagnostic and therapeutic management. Fetal Diagn Ther 14: $102-105$

Aviles A, Neri N (2001) Hematological malignancies and pregnancy: a final report of 84 children who received chemotherapy in utero. Clin Lymphoma 2: $173-177$

Brierley JD, Rathmell AJ, Gospodarowicz MK, Sutcliffe SB, Munro A, Tsang R, Pintilie M (1998) Late effects of treatment for early-stage Hodgkin's disease. Br J Cancer 77: $1300-1310$

Fisher PM, Hancock BW (1996) Hodgkin's disease in the pregnant patient. Br J Hosp Med 56: 529-532

Frank L (2000) Epidemiology. When an entire country is a cohort. Science 287: 2398 -2399, doi:10.1126/science.287.5462.2398

Green DM, Hall B (1988) Pregnancy outcome following treatment during childhood or adolescence for Hodgkin's disease. Pediatr Hematol Oncol 5: $269-277$

Green DM, Whitton JA, Stovall M, Mertens AC, Donaldson SS, Ruymann FB, Pendergrass TW, Robison LL (2002) Pregnancy outcome of female survivors of childhood cancer: a report from the childhood cancer survivor study. Am J Obstet Gynecol 187: 1070-1080, doi: 10.1067/ mob.2002.126643

Hansen D, Møller H, Olsen J (1999) Severe periconceptional life events and the sex ratio in offspring: follow up study based on five national registers. BMJ 319: $548-549$

Holmes GE, Holmes FF (1978) Pregnancy outcome of patients treated for Hodgkin's disease: a controlled study. Cancer 41: 1317-1322

Janov AJ, Anderson J, Cella DF, Zuckerman E, Kornblith AB, Holland JC, Kantor AF, Li FP, Henderson E, Weiss RB (1992) Pregnancy outcome in survivors of advanced Hodgkin disease. Cancer 70: 688-692

Knudsen LB, Olsen J (1998) The Danish medical birth registry. Dan Med Bull 45: $320-323$

Koren G, Lishner M, Zemlickis D (1996) Cancer in pregnancy: identification of unanswered questions on maternal and fetal risks. In Cancer in Pregnancy: Maternal and Fetal Risks, Koren G, Lishner M, Farine D (eds) pp 3-14. Cambridge: Cambridge University Press

Kristensen J, Langhoff-Roos J, Skovgaard LT, Kristensen FB (1996) Validation of the Danish birth registration. J Clin Epidemiol 49: $893-897$
Dannins Foundation for Scientific Research; Fritz, Georg and Marie Cecilie Glud's Foundation; and from Else and Mogens Wedell-Wedellsborgs Foundation.
Larsen H, Nielsen GL, Bendsen J, Flint C, Olsen J, Sørensen HT (2003) Predictive value and completeness of the registration of congenital abnormalities in three Danish population-based registries. Scand J Public Health 31: 12 - 16, doi: 10.1080/14034940210134194

Lishner M, Zemlickis D, Degendorfer P, Panzarella T, Sutcliffe SB, Koren G (1992) Maternal and foetal outcome following Hodgkin's disease in pregnancy. Br J Cancer 65: 114-117

Mckeen EA, Mulvihill JJ, Rosner F, Zarrabi MH (1979) Pregnancy outcome in Hodgkin's disease. Lancet 2: 590

Melbye M, Adami H-O (2002) Hodgkin's lymphoma. In Textbook of Cancer Epidemiology, Adami H-O, Hunter D, Trichopoulos D (eds) pp 520-534. New York: Oxford University Press Inc.

Mitchell AA (2000) Special considerations in studies of drug-induced birth defects. In Pharmacoepidemiology, Strom BL (ed) pp 749-763. Chichester: Wiley

Nagarajan R, Robison LL (2005) Pregnancy outcomes in survivors of childhood cancer. J Natl Cancer Inst Monogr 34: 72-76, doi: 10.1093/ jncimonographs/lgi020

Pinborg A, Loft A, Nyboe AA (2004) Neonatal outcome in a Danish national cohort of 8602 children born after in vitro fertilization or intracytoplasmic sperm injection: the role of twin pregnancy. Acta Obstet Gynecol Scand 83: $1071-1078$

Ross L, Johansen C, Dalton SO, Mellemkjaer L, Thomassen LH, Mortensen $\mathrm{PB}$, Olsen JH (2003) Psychiatric hospitalizations among survivors of cancer in childhood or adolescence. $N$ Engl J Med 349: 650-657

Signorello LB, Cohen SS, Bosetti C, Stovall M, Kasper CE, Weathers RE Whitton JA, Green DM, Donaldson SS, Mertens AC, Robison LL, Boice Jr JD (2006) Female survivors of childhood cancer: preterm birth and low birth weight among their children. J Natl Cancer Inst 98: 1453-1461, doi:10.1093/jnci/djj394

Smith LH, Dalrymple JL, Leiserowitz GS, Danielsen B, Gilbert WM (2001) Obstetrical deliveries associated with maternal malignancy in California, 1992 through 1997. Am J Obstet Gynecol 184: 1504-1512

Storm HH, Michelsen EV, Clemmensen IH, Pihl J (1997) The Danish cancer registry - history, content, quality and use. Dan Med Bull 44: 535-539

Swerdlow AJ, Jacobs PA, Marks A, Maher EJ, Young T, Barber JC, Vaughan HG (1996) Fertility, reproductive outcomes, and health of offspring, of patients treated for Hodgkin's disease: an investigation including chromosome examinations. Br J Cancer 74: 291-296

Woo SY, Fuller LM, Cundiff JH, Bondy ML, Hagemeister FB, McLaughlin P, Velasquez WS, Swan Jr F, Rodriguez MA, Cabanillas F (1992) Radiotherapy during pregnancy for clinical stages IA-IIA Hodgkin's disease. Int J Radiat Oncol Biol Phys 23: 407-412

Yusuf RZ, Naeem R (2004) Cytogenetic abnormalities in products of conception: a relationship revisited. Am J Reprod Immunol 52: 88-96, doi:10.1111/j.1600-0897.2004.00196.x 